\title{
Pluralistic perspectives on logic: an introduction
}

\author{
Colin R. Caret ${ }^{1}$. Teresa Kouri Kissel ${ }^{2}$
}

Published online: 2 January 2020

(c) Springer Nature B.V. 2020

\section{Logic and logics}

Logical pluralism is the view that there are distinct, but equally good logics. Recent years have witnessed a sharp upswing of interest in this view, resulting in an impressive literature. We only expect this trend to continue in the future. More than one commentator has, however, expressed exasperation at the view: what can it mean to be a pluralist about logic of all things? [see, e.g., Eklund (2017); Goddu (2002); Keefe (2014)]. In this introduction, we aim to set out the basic pluralist position, identify some issues over which pluralists disagree amongst themselves, and highlight the topics at the heart of the ongoing debate.

Logic qua field of study is concerned with a special relation between propositions called logical consequence or logical entailment. ${ }^{1}$ When the premises of an argument entail its conclusion, in this way, the argument is said to be logically valid. This relation may serve the role of preserving truth or it may serve an epistemic role such as grounding proof. Leaving aside such details for the time being, let us just say that logic is about an important relation-consequence or entailment-delineated by its distinctive role. Logics qua formal systems can be understood as theories of logical consequence. Logics may disagree with one another regarding the nature or the extension of logical consequence, or both.

Say that a good logic 'gets it right' in all the ways that such a theory can be measured, i.e. it adequately describes the relation at the heart of (the field of) logic. A traditional assumption, running from Aristotle to Frege, is that there is exactly one good logic in this sense. This position, known as logical monism, says that there is ultimately one privileged account of logical consequence that is superior to any alternative.

Contrary to monism, logical pluralism is the view that there is more than one equally good logic. Avowed pluralists typically do not intend the so-called 'plurality'

\footnotetext{
1 For now we will assume without comment that propositions are the right things to stand in this relation, though see the next section for potential qualifications to this assumption.
}

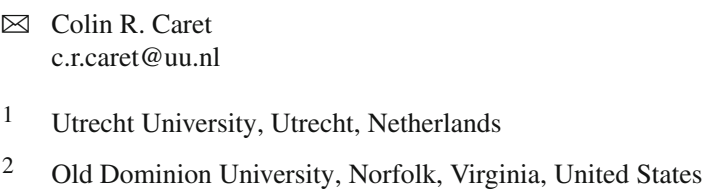


to be absolutely unrestricted, i.e. not just 'anything goes' when it comes to logic. Most pluralists agree that some theories or formal systems are clearly not candidates to play the intended role of logic. The pluralist does, however, endorse a variety of logics that she takes to be in equally good standing. This is the core commitment shared by logical pluralists. In what follows, we will describe the most influential versions of logical pluralism and say a little bit about what drives those views and what challenges they face. We will then summarize how the contributions to this special issue expand our understanding of these issues.

\section{Three milestones}

The idea of logical pluralism is often traced back to Carnap (1937), whose pluralistic outlook arose from distinctive commitments regarding the nature of logic and language. Jumping ahead several decades, the views of Beall and Restall (2006) and Shapiro (2014) have recently stimulated a lot of discussion. We present an overview of these three positions and summarize a few other important contributions to the overall pluralist dialectic.

\subsection{Carnapian conventionalism}

Carnap holds that there is no one right logic because the question 'which logic is right?' is illegitimate in a particular way. Carnap's position is pluralistic in the sense that, though he denies that it is coherent to ask which logic is correct, he does countenance the possibility of different logics being useful. A choice of logical consequence relation can only be made relative to a choice of linguistic framework, that is, "a system of...ways of speaking, subject to...rules" (Carnap 1983, p. 242).

Carnap's position can be summarized by two often-quoted passages:

Our attitude towards...requirements...[of a logic] is given a general formulation in the Principle of Tolerance: It is not our business to set up prohibitions but to arrive at conventions.

In logic, there are no morals. Everyone is at liberty to build up his own logic...All that is required of him is that, if he wishes to discuss it, he must state his methods clearly, and give syntactical rules instead of philosophical arguments.

Carnap (1937, p. 51/2)

We find that for Carnap, what is most important is not the intrinsic features of a logical system but whether the system can be explicated by syntactical rules and suits the purpose it was meant to be used for. This is a highly pragmatic approach. Here, "logical syntax is the same thing as the construction and manipulation of a calculus" (Carnap 1937, p. 5), and since we can construct different calculi depending on the language, different logics may result.

Whether a logic is correct outside of a given linguistic framework is not a wellformed question on this picture. Questions about logical correctness can only be asked relative to a linguistic framework. For Carnap, this makes the question of which 
logic is the one true logic a pseudo-question because it asks something external to a given framework - about what is really correct-when we can only meaningfully ask whether a logic (or account of logical consequence) is correct internal to a linguistic framework. The only grounds we have to accept or reject a given framework/logic pair is its efficiency as an instrument (see Carnap 1983, p. 257) and whether it accomplishes the goals we intend it to.

On the Carnapian view, we thus see an emphasis placed upon language as the basis of logic, the conventional nature of language, and practical reason as providing the grounds for linguistic/logical commitments.

\subsection{Beall \& Restall on unsettled concepts}

Beall and Restall (2000, 2006) arrive at pluralism via a more heavy-handed philosophical analysis of logical concepts. According to Beall and Restall, the concept of logical consequence is unsettled in a distinctive way: it constrains without uniquely determining its extension. They argue that the concept of logical consequence consists in a schematic expression of the platitude that logic is truth-preserving, known as the Generalized Tarski Thesis (GTT).

(GTT) An argument is valid $x$ if and only if, in every case a $_{x}$ in which the premises are true, so is the conclusion (Beall and Restall 2006, p. 29).

They also hold that cases partition into disjoint types characterized by different structural properties. The plurality of types of cases results in more than one instance of the concept. Anyone who accepts the GTT analysis of logical consequence along with the view that cases partition into types will end up being a logical pluralist of some stripe.

In particular, Beall and Restall endorse three types of cases which they refer to as possible worlds, situations, and constructions. Since, e.g., exactly one of $P$ or $\neg P$ is true in each possible world, they endorse one consequence relation in which the principle of excluded middle is valid (classical consequence). On the other hand, since there are constructions in which neither $P$ nor $\neg P$ is true, they endorse another consequence relation in which the principle of excluded middle is invalid (intuitionistic consequence).

Notably, on this view, logic is not determined by language and pluralism does not arise from variance in language. There are meant to be several equally good accounts of logical consequence over exactly the same arguments formulated in the same language. The above example of excluded middle illustrates this point: according to Beall and Restall, excluded middle is both valid (classically) and invalid (intuitionistically) and there is nothing more to say about its logical credentials. Significantly, this entails that logical terms have a 'core' meaning in all of their uses but one that fails to determine a unique inferential role.

On the Beall-Restall view, emphasis is thus placed upon how the unsettled concept logical consequence is instantiated by multiple relations, where this plurality of admissible relations arises independently of one's background language (it is perhaps best understood as arising from a metaphysically irreducible plurality of so-called cases). 


\subsection{Shapiro on mathematical pluralism}

A more recent pluralist position, staked out by Shapiro (2011, 2014), draws inspiration primarily from the richness of mathematics. Shapiro motivates his version of logical consequence from the Hilbertian perspective that "consistency is the only formal, mathematical requirement on legitimate theories" (Shapiro 2014, p. 88). Since consistency itself is defined relative to a logical consequence relation, if we relax what counts as a logical consequence relation from something monistic (ex: only the classical definition of consequence counts) to something pluralistic, we can generate more consistent and legitimate theories.

For Shapiro, logical consequence, at least as it is used for mathematics, holds relative to structure (where a mathematical structure is anything characterized by a consistent mathematical axiomatization). Because the axioms of both classical and constructive analysis systems are consistent relative to classical and intuitionistic logical consequence, respectively, they generate different structures. Each structure calls for a different logic, a logic that holds relative to that particular structure.

Shapiro makes a similar move with natural language. Shapiro suggests that "a formal language is a mathematical model of natural language in roughly the same sense as, say, a collection of point masses is a model of a system of physical objects, a set of differential equations is a model of the growth of bacteria in lakes and streams, and a Turing machine is a model of an algorithm or computing device" (Shapiro 2011, p. 537). On this conception, each conversational context (where conversational contexts include at least mathematical structures, as described above) determines the correct logic. Logic is relative to context.

Since Shapiro endorses the Hilbertian perspective, and also that classical analysis and intuitionistic analysis are legitimate mathematical systems, Shapiro's view is a logical pluralism. The rules by which mathematicians reason in those two systems are different: they are classical in the first case, and intuitionistic in the second. Thus, both classical and intuitionistic logics are legitimate logics.

Further, Shapiro claims that

For some purposes...it makes sense to say that the classical connectives and quantifiers have different meanings than their counterparts in intuitionistic, paraconsistent, quantum, etc. systems. In other situations, it makes sense to say that the meaning of the logical terminology is the same in the different systems. Shapiro (2014, p. 127)

So, contra Beall and Restall (2006), Shapiro does take it that his pluralism has an effect on connective meanings. In particular, they are much more variable than we might have thought. Sometimes two mathematicians using different logics will use the same connectives, and sometimes they will not.

On the Shapiro view, emphasis is placed on the use of logic in mathematics, the diversity of logical consequence relations that can generate mathematical structures, and a flexible notion of when two connectives share a meaning. 


\subsection{Other strands of pluralism}

As this debate is attracting more and more attention, so too is it attracting more and more proponents. Varzi (2002), for example, defends a pluralistic view of logic inspired by Carnap and Tarski. He summarized his position as Tarskian Relativism (TR):

(TR) Logical validity is relative to a choice of logical terms and there is no principled distinction between logical and non-logical terms.

Haack (1974) proposes that there are needs classical logic cannot fill, and thus we must look for different logical consequence relations. Russell (2008) proposes that the right logic is relative to the choice of truth bearers (this would change the default we allude to in footnote ). Field (2009) suggests that logic is relative to the set of norms in play, and Cook (2010) thinks it is relative to how we choose to idealize natural language. The list goes on. ${ }^{2}$

\section{Monism strikes back}

Because pluralism has garnered such attention in recent years, it has also been subject to a number of objections. Of importance for this special issue are two in particular: what we will call the meaning-variance worry, and the collapse problem.

\subsection{The meaning-variance worry}

For Quine (1986), logical monism must be true. Moreover, given the meanings Quine attaches to the logical connectives and his insistence that those are the only logical connectives, disagreements over which logic is correct are merely verbal. Though he is a monist and attaches a particular meaning to the connectives, Quine's meaning variance worry suggests a more general problem for pluralists. It must be possible for pluralists to talk about the relationships between logics, and to communicate even if they are "speaking different logical languages". Otherwise all debate about which logic is best will be merely verbal dispute, since people engaged in the discussion will just be talking past each other.

It is easy to see where things could go wrong for the pluralist. Suppose the classical connectives are defined by truth conditions, and the intuitionistic connectives defined by inferential role. Now, imagine two people having a debate about whether the law of excluded middle, " $P \vee \neg P$ ", is valid. One is associating truth conditional meanings to " $\vee$ " and " $\neg$ ", and the other inferential roles. So, when one of our participants utters "the law of excluded middle is valid" and the other says "nope, that's not right", they may think they are having a substantive debate, but cannot be. This is because they

\footnotetext{
2 The list goes so far as to include things like logical nihilism, the position that there is no correct logic. We will not address that here, but see see Russell's "An Introduction to Logical Nihilism", forthcoming in Proceedings of the 15th International Congress, for a defense. Other signifcant contributions to the pluralism debate include Bremer (2014), Griffiths (2013), Hjortland (2013), Pedersen and Wright (2013), Priest (2014), and Restall (2002).
} 
have assigned different meanings to " $\vee$ ” and “ $\neg$ ”, and so are talking about different laws! If Quine is right here, and connectives in different logics must have different meanings, then we cannot have any sort of cross logical debate. Pluralists like Beall and Restall (2006) or Shapiro (2014) must say something about this problem because an explicit part of those views is that (at least sometimes) there are equally good logics that involve the same connectives.

\subsection{The collapse problem}

Keefe (2014), Priest (2008) and Read (2006) have separately articulated an argument against the kind of pluralism defended by Beall and Restall (2006). Two features of Beall and Restall's view drive this problem. First, they hold logic to be intrinsically normative. This rules out some formal systems as unsuited to the role of logic. Second, Beall and Restall take logic to be topic neutral. That is, variation in logic is not tied to variation in language. Rather, the plurality of logics are meant to normatively govern the exact same set of arguments in exactly the same language. As a result, on Beall and Restall's view, if an argument is valid in any legitimate logic, then any argument in that form is valid and, thus, reason-guiding.

A simple version of the Keefe-Priest-Read argument runs as follows. Since Beall and Restall only endorse classical logic and a couple of sub-classical logics, the strongest logic which applies in any situation will be classical logic. On such a view, we always should reason by classical logic if we want to gain the most information. ${ }^{3}$ But if our reasoning should always be guided by classical logic, then it does not seem like there is much of a role to play for the other, sub-classical, logics which Beall and Restall claim are legitimate. So, Keefe, Priest and Read concludes that pluralistic views of the kind articulated by Beall and Restall are implicitly committed to logical monism, with classical logic as the one true logic. This is more than just a challenge for Beall and Restall, too. Any logical pluralism who holds that logic is normative and topic neutral will have to face it.

\section{Summary of contributions}

This issue contains papers which address several of the above themes. We have grouped them into four categories, though they can be read in any order. The first set of papers address logical generality and relativity, and issues with metalanguages. The second address issues with admissibility (in the sense of the logics accepted into the pluralistic system), normativity and the use of logic. The third set deal with logical pluralism's relationship to other theories. Finally, we have papers which consider logical rivalry and the individuation of logics.

\footnotetext{
3 This is for the following reason. Suppose we have the information that all members of set $\Sigma$ are true. Take any shared argument form $\Sigma \vdash A$. On Beall and Restall's view, if this form is valid in any logic they endorse, it is valid in classical logic. So, we never go wrong by drawing the conclusion $A$, but we can also draw more conclusions by using classical inferences that are not valid in the other logics of the plurality. Thus, classical reasoning is always the superior method of gaining information by deduction.
} 


\subsection{Generality, relativity, and metalanguages}

The most prominent logical pluralists accept that logical connectives are meaninginvariant across distinct logics (at least in some qualified form). The very possibility of meaning-invariance across logics has been scrutinized by Quine on the basis of his monistic view of logic. Is meaning-invariance compatible with a view of logic as entirely general? Does it require logics to each be relativized in some way? If logics are domain-relative, where can the pluralist 'stand' (as it were) in order to allow them to endorse multiple logics? What kind of meta-language allows this? Several of our contributors address these pressing questions.

Kouri Kissel's paper focuses on what happens when people try to communicate with each other, if pluralism is true. On some pluralisms, notably domain-specific logical pluralisms, people can be engaged in the same project while using different logics, or using different logical connectives. Initially, it would seem that anyone involved in such a project would not be able to communicate (or disagree) with anyone else involved in such a project, as they would be "speaking different logical languages". Kouri Kissel's paper proposes interpreting such exchanges as meta-linguistic negotiations rather than merely verbal disputes. Thus, the people involved in those exchanges are in fact able to communicate with each other.

Wyatt and Payette argue that logic is not general, in the sense that the rules and principles associated with a logical system are not universally applicable. They call this "logical generalism", and contrast it with their preferred view, which they dub "logical particularism". If we are to be logical generalists, we must hold that logical laws have no counterexamples, and are always valid. But, claim Wyatt and Payette, this is not the case. Thus, if we want to be generalists, we must be nihilists. To avoid nihilism, they adopt logical particularism, the view that the laws of logic are not universal. They argue that this position makes logic more akin to a science, which they hold to be an advantage. An upshot of this view is that logic systems are primarily explanatory, just like theories in science.

Golan's paper argues that there is no available meta-language in which we can have meaningful debates about logic if logical pluralism is true. He argues that meaninginvariant pluralists need a meta-language which is suitably neutral, so that it doesn't force us to adopt its norms rather than those of our object-level logics. Further, Golan holds that this neutrality can come in two forms: it either must contain a collection of agreed upon inferences, or it must be neutral-as-such. Neither of these options work, he claims; both run afoul of Quine's claim that when we change the logic, we change the meanings of the connectives. The first does, since the agreed upon inferences will require connectives with the same meanings, and so we will need to fix the meanings of the connectives in all of our logics. The second results in a type of collapse [a la Keefe (2014) and Caret (2017)], since the neutral meta-language will impose the meanings of its connectives on all the object-language logical connectives.

\subsection{Admissibility, normativity, and use}

Logical pluralists often want to limit the range of candidate logics by imposing some kind of admissibility criteria. As noted above, pluralists do not usually believe that 
'anything goes' and one way to get this across is to insist that some theories or formal systems are clearly not candidates to play the intended role of logic. But the issue of exactly how logical pluralists should finesse their view about the admissibility criteria for logics has not been very widely discussed. Should truth-conduciveness or epistemic normativity be amongst these criteria? The collapse problem indicates that these may be problematic criteria. Does inferential use or adoptability restrict the range of candidate logics? Several of our contributors address these pressing questions.

Logical normativism is the view that logic is intrinsically normative. Blake-Turner and Russell argue that logical pluralists need not and should not be logical normativists. They contend that logic qua field of study describes a static relation of truth-preservation in the first instance (cf. Harman 1986). We often think of logic as a normative discipline because background epistemic norms together with the 'logical facts' have consequences for what we ought to believe. Blake-Turner and Russell argue, however, that such normativity is extrinsic to and derivative of logic itself. The greatest strength of being a non-normativist pluralist is that such a views seems immune to the threat of the collapse problem. However, they acknowledge that pluralists such as Beall and Restall have good reason to be normativists: for Beall and Restall, normativity acts as a criterion for what count as admissible logics. In response, Blake-Turner and Russell suggest that it is possible to accept entirely non-normativist criteria of admissibility. For example, a pluralist might only recognize logics as being 'legitimate' if they are useful for some application, where such pragmatic criteria are distinguished from the view that it is in the nature of logic itself to be useful.

Ferrari and Orlandelli bring a proof-theoretic perspective to bear on meaninginvariant logical pluralism. They first describe a proof-theoretic framework in which classical and intuitionistic logics coexist (cf. Restall 2014). Ferrari and Orlandelli argue that it is perfectly coherent for the pluralist to endorse this framework and, as a result, to treat single- and multiple-conclusion calculi as equally legitimate. They then turn to a problem raised by Kouri (2016). Every logical pluralist needs to have criteria for admissible logics, but for a pluralist with a proof-theoretic perspective, some criteria have unappealing results (e.g. a criterion of truth-preservation would rule out relevance logics within a proof-theoretic framework). In response to this worry, Ferrari and Orlandelli suggest an approach that is more more fully proof-theoretic in spirit. They suggest that Belnap's notion of harmony is a plausible criterion of admissibility for the proof-theoretic pluralist, and that it is compatible with endorsing relevance logics. They conclude with a discussion of how proof-theoretic pluralists should understand the notions of necessity and formality in connection with logic.

Finn argues that considerations of adoptability imply that there are limitations to any possible version of logical pluralism. Kripke's Adoption Problem is about whether and how a logical agent can chose to adhere to rules of inference that they were not previously disposed to adhere to, in short: could I adopt new logical rules? Finn claims that the upshot of this problem is that some logics are inadequate candidates for adoption. Rules such a modus ponens (MP) and universal instantiation (UI) are unadoptable insofar as we could not learn how to follow them unless we already follow them. But why is this the case? Finn goes beyond Kripke at this point to offer her own analysis that unadoptable rules are special because they are 'self-governing'. All logical rules have a universal and conditional form, which means that when we 
state the rules of UI and MP these rules play a role in governing themselves - because adhering to UI and MP is a prerequisite for understanding any logical rule. The lesson for the logical pluralist, then, is that there can only be equally good logical systems up to a point. If legitimate logics are all adoptable logics, then pluralism must be limited to systems with adequate meta-logics.

\subsection{Inter-related pluralisms}

Outside of logic, there are many other topics in the philosophical literature where philosophers advocate pluralistic views: in metaphysics, epistemology, truth studies, the philosophy of science and mathematics just to name a few of the most relevant views. Is logical pluralism related to these other pluralistic views? Does our concept of truth or best mathematical practice provide any support for a version of logical pluralism? (cf. Pedersen 2014) Several of our contributors address these pressing questions.

Shapiro and Lynch's paper tries to assess whether, to be a logical pluralist, you must be a truth pluralist, or vice versa. They focus their efforts on the relationship between Shapiro's pluralism about logic, and Lynch's pluralism about truth. The answer they settle on is that there is an obvious way in which logic and truth are tied, but whether being a pluralist of one stripe forces you to be a pluralist of the other is a subtle and complex issue, and depends on how we settle a myriad of questions (including things like what defines the meanings of the logical connectives, what truth properties we are considering, etc.).

Priest scrutinizes the relationship between pluralistic views of mathematics and logic. Some recent logical pluralists (notably, Shapiro (2014)) draw a tight connection between the two topics. Priest argues that this connection is not so tight. He articulates a type of mathematical pluralism that revolves around mathematical structure. Priest happily agrees that mathematical studies of what is true-in-a-structure do employ diverse logics in an important way: intuitionistic analysis relies essentially on the failure of excluded middle, paraconsistent topology relies essentially on the failure of ex falso quodlibet. In this respect, Priest is a mathematical pluralist. He resists the move to logical pluralism, however, because logical consequence is about preservation of truth (simpliciter). Whereas truth-in-a-structure is a relativized property, loosely analogous to truth-in-a-story, the property of truth (simpliciter) is not relativized to anything (story, structure, etc.). Priest contends that the monolithic nature of truth (simpliciter) best fits with a monistic view of logic. More narrowly, he simply points out that his form of mathematical pluralism, which accepts that truth-in-a-structure is preserved in diverse ways, does not entail logical pluralism, i.e. the view that truth (simpliciter) is preserved in diverse ways.

Caret offers a meta-analysis of topics and themes in the logical pluralism literature. His stated goal is to better understand what is at stake in the debate and how we could garner evidence in support of a pluralistic view of logic. Caret argues that the most interesting versions of pluralism are those that are 'properly' pluralistic, meaning that they hold some core logical relation (e.g. confirmation, entailment, consistency) to be irreducibly fragmentary. He criticizes the way that some pluralists have relied heavily on untestable intuitions to motivate their views and provides a case study of Shapiro's 
recent work on logical pluralism, which improves on this situation. Caret argues that Shapiro's appeal to evidence grounded in mathematical practice is compelling because it prompts an abductive question to which logical pluralism is an obvious candidate answer. Caret then describes how this methodology can be generalized. The point is not to show that logical pluralism is correct (or incorrect), but to simply provide a better understanding of how we can conduct a substantive, naturalistically respectable debate about logical pluralism.

\subsection{Logical individuation and rivalry}

Another important topic is how logical pluralists should individuate logics and conceive of logics as 'rivals' in a way that allows them to say that they genuine endorse multiple 'rival' logics. It may seem prima facie obvious how to approach this issue, but some of our contributors raise unforeseen problems and offer novel ideas about how to improve upon the standard views of logical individuation and rivalry.

French's paper argues that the Built-In Opponent conception of deduction (Dutilh Novaes 2015), Dutilh Novaes and French (2018) generates an interesting form of logical pluralism. This conception of deduction requires that proofs are explanatory, and can give rise to a pluralism depending on the restrictions placed on what counts as an explanatory proof. It relies on a dialogical understanding of deduction, where deductions are a special kind of dialogue, where, loosely, a "skeptic" seeks reasons that a proposition follows from a set of propositions from a "prover". An argument is valid if the prover can convince the skeptic that the proposition follows from the premises on all ways that the skeptic makes their case. The reasons the prover gives are meant to convince the particular audience (the skeptic) that the proposition follows from the premises. And this is where pluralism sneaks in: what is convincing to one audience might not be convincing to another, and so the logical validities will depend on the audience.

Barrio, Pailos, and Szmuc investigate how assumptions about logical rivalry have played a role in the collapse problem for logical pluralism. The standard Collapse Argument makes the following dubious assumption: that logics are rivals just when there is an inference that is valid in one logic but not the other. Barrio, Pailos, and Szmuc show that this is faulty. Some substructural logics have the same set of valid inferences as classical logic, but they disagree with classical logic at the level of metainferences. This is a kind of rivalry, but one that falls outside the usual scope of logical pluralism. However, it is not enough to say that logics are rivals just when there is a meta-inference that is valid in one logic but not the other. In fact, some substructural logics have the same sets of valid inferences and meta-inferences as classical logic, but they disagree with classical logic at the level of meta-meta-inferences. Barrio, Pailos, and Szmuc argue that we should define rivalry of logics by generalizing across all levels of meta-inferences, i.e. logics are rivals just when they agree up to some level, but disagree beyond that. 


\section{Concluding thoughts}

This collection of papers is far from the final word on logical pluralism. We foresee a number of important questions with which future research on logical pluralism must contend. How do we define and individuate logics? Is the important 'core subject' of logic best analyzed in terms of truth, epistemic role, or something else entirely? When there are indefinitely many prima facie viable logics, how do we settle the question of 'admissibility criteria'? Is logical pluralism related to other forms of indeterminacy and vagueness or is it another beast entirely? As a scientific community, can we seek consensus and do we need to seek consensus about logical matters? Does replicable empirical data bear on these questions and, if so, how? This special issue helps to clarify and advance certain aspects of this debate, but there is plenty of room for further investigation. We hope that these papers will not only offer valuable insights on their own, but also point the way forward for increasingly fruitful discussions of logical pluralism.

\section{References}

Beall, J., \& Restall, G. (2000). Logical pluralism. Australasian Journal of Philosophy, 78(4), 475-493.

Beall, J., \& Restall, G. (2006). Logical pluralism. Oxford: Oxford University Press.

Bremer, M. (2014). Restall and Beall on logical pluralism: A critique. Erkenntnis, 79(2), 293-299.

Caret, C. R. (2017). The collapse of logical pluralism has been greatly exaggerated. Erkenntnis, 82, 739-760.

Carnap, R. (1937). The logical syntax of language. Harcourt: Brace and Company.

Carnap, R. (1983). Empiricism, semantics and ontology. In P. Benacerraf \& H. Putnam (Eds.), Philosophy of mathematics (pp. 241-257). Cambridge: Cambridge University Press.

Cook, R. T. (2010). Let a thousand flowers bloom: A tour of logical pluralism. Philosophy Compass, 5(6), 492-504.

Dutilh Novaes, C. (2015). A dialogical, multi-agent account of the normativity of logic. Dialectica, 69, 587-609.

Dutilh Novaes, C., \& French, R. (2018). Paradoxes and structural rules from a dialogical perspective. Philosophical Issues, 28, 129-158.

Eklund, M. (2017). Making sense of logical pluralism. Inquiry, online first 2017.

Field, H. (2009). Pluralism in logic. The Review of Symbolic Logic, 2(2), 342-359.

Goddu, G. C. (2002). What exactly is logical pluralism? Australasian Journal of Philosophy, 80(2), 218230.

Griffiths, O. (2013). Problems for logical pluralism. History and Philosophy of Logic, 34(2), 170-182.

Haack, S. (1974). Deviant logic. Cambridge: Cambridge University Press.

Harman, G. (1986). Change in view: Principles of reasoning. Cambridge: MIT Press.

Hjortland, O. T. (2013). Logical pluralism, meaning-variance, and verbal disputes. Australasian Journal of Philosophy, 91(2), 355-373.

Keefe, R. (2014). What logical pluralism cannot be. Synthese, 191(7), 1375-1390.

Kouri, T. (2016). Restall's proof-theoretic pluralism and relevance logic. Erkenntnis, 81(6), 1243-1252.

Pedersen, N. J. L. L. (2014). Pluralism × 3: Truth, logic, metaphysics. Erkenntnis, 79(2), 259-277.

Pedersen, N. J. L. L., \& Wright, C. (2013). Truth and pluralism. Oxford: Oxford University Press.

Priest, G. (2008). Doubt truth to be a liar. Oxford: Oxford University Press.

Priest, G. (2014). Logical pluralism: Another application for chunk and permeate. Erkenntnis, 79(2), 331338.

Quine, W. V. O. (1986). Philosophy of logic (2nd ed.). Cambridge: Harvard University Press.

Read, S. (2006). Monism: The one true logic. In D. DeVidi \& T. Kenyon (Eds.), A logical approach to philosophy: Essays in honour of Graham Solomon (pp. 193-209). The Netherlands: Springer.

Restall, G. (2002). Carnap's tolerance, meaning, and logical pluralism. Journal of Philosophy, 99(8), 426443. 
Restall, G. (2014). Pluralism and proofs. Erkenntnis, 79, 279-291.

Russell, G. (2008). One true logic? Journal of Philosophical Logic, 37, 593-611.

Shapiro, S. (2011). Varieties of pluralism and relativism for logic. In S. D. Hales (Ed.), A companion to relativism (pp. 524-552). New York: Wiley.

Shapiro, S. (2014). Varieties of logic. Oxford: Oxford University Press.

Varzi, A. C. (2002). On logical relativity. Philosophical Issues, 12(1), 197-219.

Publisher's Note Springer Nature remains neutral with regard to jurisdictional claims in published maps and institutional affiliations. 